\title{
Spectral Characterisation of Spontaneous Parametric-Down Conversion Entangled Photons Source in Femtosecond Pulsed Regime
}

\author{
G. Brida, V. Caricato, M. Genovese, M. Gramegna \\ Optics Division \\ INRiM - Istituto Nazionale di Ricerca Metrologica \\ Turin, Italy \\ m.gramegna@inrim.it
}

\author{
M. V. Fedorov \\ A. M. Prokhorov General Physics Institute, \\ Russian Academy of Sciences \\ Moscow, Russia \\ fedorovmv@gmail.com
}

\author{
S. P. Kulik \\ Faculty of Physics \\ Moscow State University \\ Moscow, Russia \\ sergei.kulik@gmail.com
}

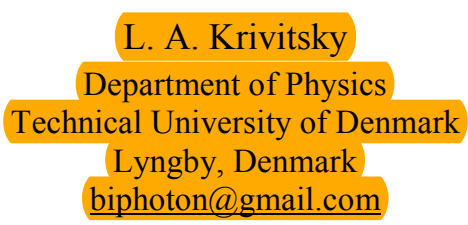

Actually, the continuous development of new quantum techniques enables to explore always deeper the field of twophoton correlation measurements and all what concernes about the fundamental properties of two-photon correlation function. In particular, biphoton states can be characterized with respect to both discrete than continuous variables, where the latter case is far from being fully investigated, especially in the short-pulse pump regime.

In this work the interest is focused on the study of the degree of entanglement with respect to the continuous frequency variables and the entanglement-induced wavepacket narrowing of biphoton states, when the SPDC pump has the form of a sequence of short pulses (femtoseconds regime), and at given polarizations and propagation directions of signal and idler photons. In such a case the pump spectrum is relatively wide and it is possibile to consider the frequencies of the signal and idler photons as additional variables characterizing SPDC biphoton states [4$6]$.

The entanglement degree of a two-particle wave function has been evaluated by the parameter $\boldsymbol{R}_{\omega}$, representing the ratio between the entanglement-free wave-packet width distribution and the coincidence one. This represents the effective degree of entanglement when it is large and, as the preliminary experimental measurements show, the achievable entanglement of biphoton states can be very high with a large contrast between coincidence and singles distributions.

\section{THEORETICAL ASSUMPTIONS}

The present experimental work relies on the theoretical proposal [4] aiming to demonstrate that when pairs of correlated photons are generated through SPDC by a train of short pulses (femtosecond regime), it is possible to measure 
the degree of entanglement through frequency variables and show that the achievable entanglement of biphoton states can be very high. The physical reason of an high entanglement relies on the temporal walk-off, i.e., slower propagation in a crystal of the pump photons compared to the emitted ones.

Moreover, the theoretical assumptions consider a SPDC spectrum relatively wide, a wide-crystal approximation, and a Gaussian shape for the Fourier transformed pump field strength. The working conditions considered a 'e $->0+0$ ' SPDC decay, with a purely collinear propagation directions of signal and idler photons.

In the framework of these assumptions and approximations, the expression for the frequency-dependent biphoton wave function results to be:

$$
\Psi\left(\omega_{1}, \omega_{2}\right) \propto E_{p}\left(\omega_{1}+\omega_{2}\right) \int_{0}^{L} d z \exp \left[i \frac{z \Delta\left(\omega_{1}, \omega_{2}\right)}{c}\right]
$$

where-the-energy-conservation-rule- $=-1-+-2$-is fullfilled among pump, signal and idler beams, and the integration over $\mathrm{z}$ goes over the entire length $\boldsymbol{L}$ of the crystal along the laser axis; $\Delta\left(\omega_{1}, \omega_{2}\right)$ is the phase mismatch.

The ratio between the FWHM of the single counts and coincidence distributions, $\boldsymbol{R}_{\omega}=\Delta \omega_{s} / \Delta \omega_{\mathrm{t}}$, is considered to be a good measure of the entanglement degree for bipartite systems, and is given by equation:

$$
R_{\omega}=\frac{\Delta \omega_{1}^{(s)}}{\Delta \omega_{1}^{(c)}}=\frac{A^{3 / 2}}{2.78} \sqrt{\frac{2 \pi \ln 2}{B}} \frac{L}{\sqrt{\lambda_{0} c \tau}}
$$

where $\mathrm{A}$ and $\mathrm{B}$ are dimensionless constants related to the phase mismatch, $\boldsymbol{L}$ the crystal length, and $\boldsymbol{\tau}$ the pump pulse duration.

This parameter is approximately equal to the Schmidt number $\boldsymbol{K}$ [4, and ref. therein] which is hard to be measured directly, therefore the $\boldsymbol{R}_{\omega}$ serves as an operational entanglement measure. The greater $\boldsymbol{R}$, the higher entanglement between two photons; if $\boldsymbol{R}_{\omega}=K=1$ the state is completely separable.

The degree of entanglement increases linearly with the length of the crystal, and decreases with the growing pump pulse.

\section{EXPERIMENTAL SET-UP}

The experimental apparatus, reported in Fig. 1, plays on a femto-second Mode-Locked pulsed source pumping, in our case, a nonlinear $\mathrm{LiIO}_{3}$ crystal of $10 \mathrm{~mm}$ length, to generate frequency entangled biphoton states.

The pulse pump is supplied by a Coherent MIRA 900 Laser at a working wavelength of $795 \mathrm{~nm}$ doubled in frequency to obtain a short pulse of $\Delta \lambda_{\mathrm{p}}=1.31 \mathrm{~nm}$. This

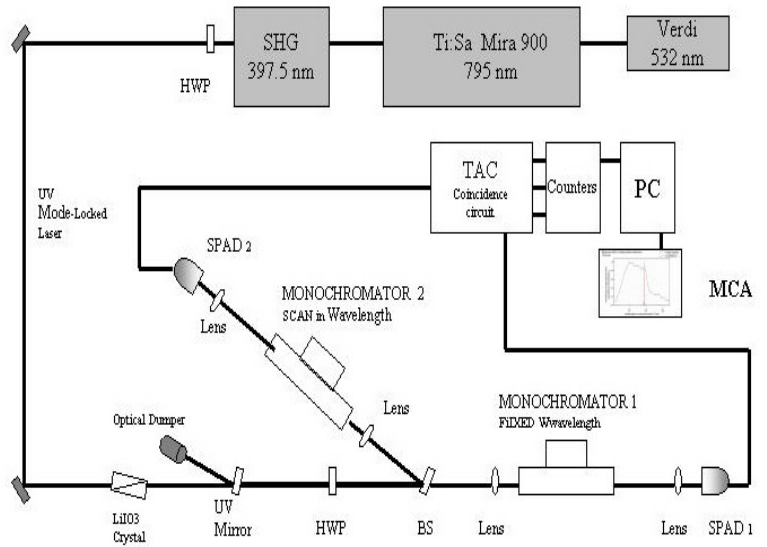

Figure 1. Sketch of the experimental set-up (HWP: Half Wave Plate; BS: Beam Splitter; SPAD: Single-photon Avalanche Diode; TAC: Time-to Amplitude converter; MCA: Multichannel Analyzer).

pump corresponds to a frequency of $\omega_{\mathrm{p}}=4.74 * 10^{15} \mathrm{~s}^{-1}$ and $\Delta \omega_{\mathrm{p}}=1.56 * 10^{13} \mathrm{~s}^{-1}$, with pulse duration $\tau_{\mathrm{p}}=177 \mathrm{fs}$.

The nonlinear interaction produces a frequency entangled state through a type I collinear degenerate SPDC, where a horizontally polarized beam hits the crystal and generates two vertically polarized photons ('e -> o + o' SPDC decay). After a beamsplitter (BS) the photons address to two singlephoton detectors (SPAD: Perkin-Elmer SPCM-AQR-15), operating in counting regime. In front of each detector it is placed a monochromator with a spectral resolution of $0.2 \mathrm{~nm}$ determined by the width of the input/output slits.

One monochromator is fixed at the central wavelength of SPDC $(795 \mathrm{~nm})$, the other one is scanned in a range around the central wavelength in order to study the spectral distribution for both single counts and coincidences.

After the detection process, the electrical pulses from the SPADs are sent to a coincidence circuit, and the temporal distribution is analyzed by a Time-to-Amplitude converter (TAC) and a Multichannel Analyzer (MCA).

\section{RESULTS AND DISCUSSION}

The preliminary experimental measurements relative to a $10 \mathrm{~mm}$ crystal, in comparison with the theoretical model [4] for a similar configuration, are reported in Table 1.

The measured coincidence distribution obtained scanning the wavelength of the monochromator in the idler branch around $\lambda_{\mathrm{c}}=795 \mathrm{~nm}$ is sketched in Fig. 2, where it is visible a peak with $\Delta \lambda_{\mathrm{c}}=0.49 \mathrm{~nm}$, corresponding to a frequency $\omega_{\mathrm{c}}=2.37$ $* 10^{15} \mathrm{~s}^{-1}$ with $\Delta \omega_{\mathrm{c}}=1.46 * 10^{12} \mathrm{~s}^{-1}$.

On the other side, the spectral distribution of single counts obtained scanning around the same central wavelength, results in a width of about $\Delta \lambda_{\mathrm{s}}=75.08 \mathrm{~nm}$, corresponding to $\omega_{\mathrm{s}}=2.37 * 10^{15} \mathrm{~s}^{-1}$ and $\Delta \omega_{\mathrm{s}}=2.24 * 10^{14} \mathrm{~s}^{-1}$.

On the consequence of these results, the evaluation of the 
TABLE I.

\begin{tabular}{|c|c|}
\hline Theoretical Model & $\begin{array}{c}\text { Preliminary Experimental } \\
\text { Results }\end{array}$ \\
\hline Pump & Pump \\
\hline$\lambda \mathrm{p}=397,5 \mathrm{~nm}$ & $\lambda \mathrm{p}=397,5 \mathrm{~nm}$ \\
\hline$\Delta \lambda \mathrm{p}=1.31 \mathrm{~nm}$ & $\Delta \lambda \mathrm{p}=1.31 \mathrm{~nm}$ \\
\hline$\omega \mathrm{p}=4.74 * 10^{15} \mathrm{~s}^{-1}$ & $\omega \mathrm{p}=4.74 * 10^{15} \mathrm{~s}^{-1}$ \\
\hline$\Delta \omega \mathrm{p}=1.56 * 10^{13} \mathrm{~s}^{-1}$ & $\Delta \omega \mathrm{p}=1.56 * 10^{13} \mathrm{~s}^{-1}$ \\
\hline$\tau p=177 \mathrm{fs}$ & $\tau p=177 \mathrm{fs}$ \\
\hline Coincidences Distribution & Coincidencse Distribution \\
\hline$\lambda \mathrm{c}=795 \mathrm{~nm}$ & $\lambda \mathrm{c}=795.6 \mathrm{~nm}$ \\
\hline$\Delta \lambda \mathrm{c}=0.315 \mathrm{~nm}$ & $\Delta \lambda \mathrm{c}=0.49 \mathrm{~nm}$ \\
\hline$\omega \mathrm{c}=2.37 * 10^{15} \mathrm{~s}^{-1}$ & $\omega \mathrm{c}=2.37 * 10^{15} \mathrm{~s}^{-1}$ \\
\hline$\Delta \omega \mathrm{c}=0.94 * 10^{12} \mathrm{~s}^{-1}$ & $\Delta \omega \mathrm{c}=1.46 * 10^{12} \mathrm{~s}^{-1}$ \\
\hline$\Delta \lambda \mathrm{p} \approx 4.15 \Delta \lambda \mathrm{c}$ & $\Delta \lambda \mathrm{p} \approx 2.66 \Delta \lambda \mathrm{c}$ \\
\hline Single Counts Distribution & Single Counts Distribution \\
\hline$\lambda \mathrm{s}=795 \mathrm{~nm}$ & $\lambda \mathrm{s}=795 \mathrm{~nm}$ \\
\hline$\Delta \lambda \mathrm{s}=102 \mathrm{~nm}$ & $\Delta \lambda \mathrm{s}=75.08 \mathrm{~nm}$ \\
\hline$\omega \mathrm{s}=2.37 * 10^{15} \mathrm{~s}^{-1}$ & $\omega \mathrm{s}=2.37 * 10^{15} \mathrm{~s}^{-1}$ \\
\hline$\Delta \omega \mathrm{s}=3.05 * 10^{14} \mathrm{~s}^{-1}$ & $\Delta \omega \mathrm{s}=2.24 * 10^{14} \mathrm{~s}^{-1}$ \\
\hline$\Delta \lambda \mathrm{s} \approx 78 \Delta \lambda \mathrm{p}$ & $\Delta \lambda \mathrm{s} \approx 57 \Delta \lambda \mathrm{p}$ \\
\hline Entanglement Degree & Entanglement Degree \\
\hline $\mathrm{R} \omega=324$ & $\mathrm{R} \omega=153$ \\
\hline
\end{tabular}

ratio between the FWHM of the coincidence and singlecounts distributions gives $\boldsymbol{R}_{\omega}=\Delta \omega_{3} / \Delta \omega_{c} \sim 153$, showing a very high degree of biphoton entanglement, being it strictly larger than 1, where $\boldsymbol{R}_{w}=1$ represents a completely separable state. The large contrast between the width of coincidence and single-particle distributions is clearly visibile in the graphycal comparison of the normalized curves reported in Fig. 3.

The experimental measurement put in evidence anyway that the value obtained for $\boldsymbol{R}_{\omega}$ results lower than theoretical estimations $\left(\boldsymbol{R}_{\omega} \sim 324\right)$ performed for the parameters of SPDC used in experiment.

The main possible sources for this discrepancy in the preliminary results may be identified in a number of possible causes: the narrow spectral width of the coincidences

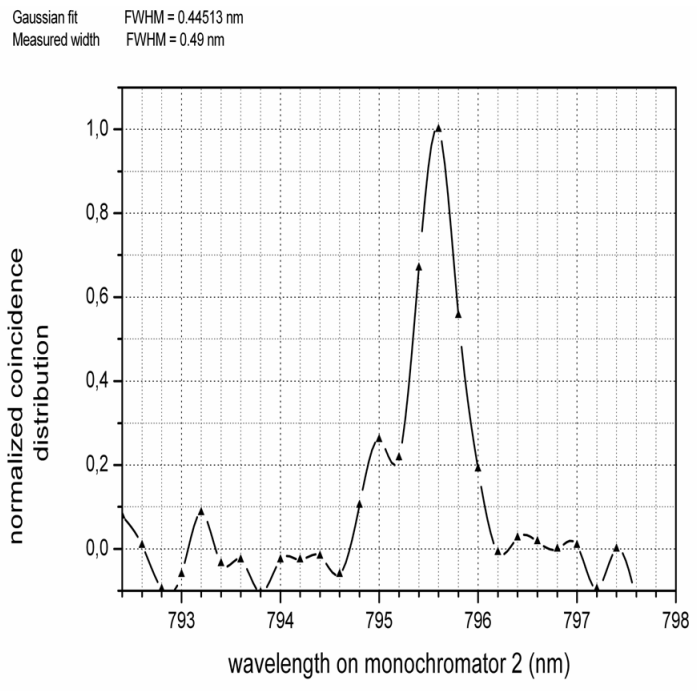

Figure 2. Coincidence distribution obtained scanning the monochromator 2 in the idler gate $(0.2 \mathrm{~nm}$ resolution).

distribution close to the spectral resolution of the monochromator, the spatial-frequency chirp of the pump pulse that takes place when the radiation propagates through the filtering prisms, causing a depletion in the SPDC decay, and not ideal optical allignment of monochromator gratings during a scan over more than $100 \mathrm{~nm}$. All these effects will be deeper investigated in a next step of the experiment.

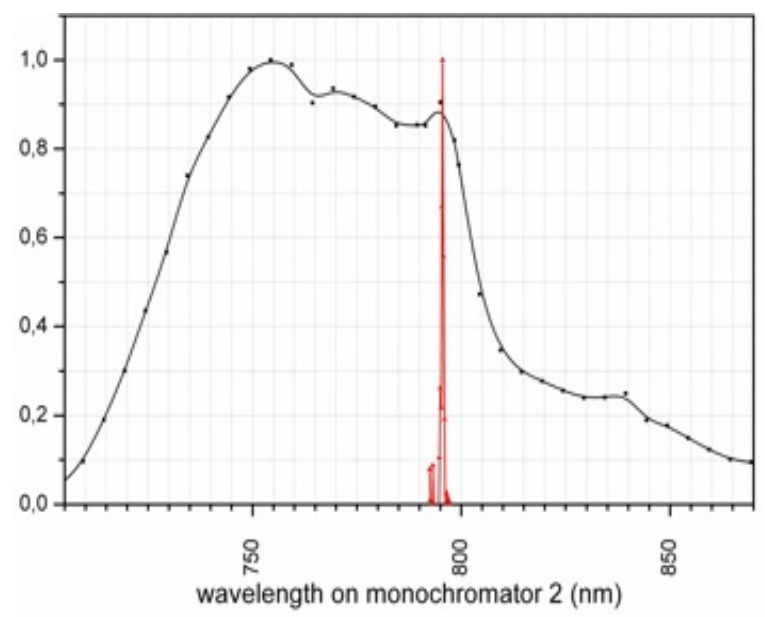

Figure 3. Single-counts distribution (FWHM $=75.08 \mathrm{~nm}$ ) versus Coincidences distribution $(\mathrm{FWHM}=0.49 \mathrm{~nm})$. 


\section{CONCLUSIONS AND Future Plans}

The experiment reported shows clear evidences for biphoton coincidence spectrum narrower and single-particle one wider than the pump spectrum, for frequency entangled states generated by type-I SPDC crystal pumped by short pulses, resulting in a high entanglement degree of SPDC biphoton states, and in strong agreement with the theoretical model.

The next step of the experiment will be devoted to the investigation of the behaviour of the $\boldsymbol{R}_{\omega}$ parameter with nonlinear crystals of different dimensions, to check the theory predictions for which the degree of entanglement increases linearly with $\boldsymbol{L}$, and the decreasing of it with the growing pump pulse.

At the same time, it will be realized an optical compressor in front of the crystal to compensate for the pulse chirping.

\section{ACKNOWLEDGMENT}

This work has been partially supported by Regione Piemonte E14 contract, San Paolo Foundation and by CNRCNISM convention.

\section{REFERENCES}

[1] D. Bouwmeester, A. K. Ekert, A. Zeilinger, "The physics of quantum information: quantum cryptography, quantum teleportation, quantum computation, Springer, New York, 2000.

[2] M. Genovese, "Research on hidden variable theories: review of recent progresses", Physics Reports, (2005), 413/6.

[3] L. Mandel, E. Wolf, "Optical Coherence and Quantum Optics", Cambridge Univ. Press, Cambridge, 1995.

[4] Yu. M. Mikhailova, P.A. Volkov, M.V.Fedorov, "Frequency and temporal entanglement of biphoton states in spontaneous parametric down conversion with a short-pulse pump", arxiv:quant-ph/0801.0689v1, (4 Jan 2008).

[5] W. P.Grice, A. B. U'Ren, I. A. Walmsey, "Eliminating Frequency and space-time correlations in multiphoton states", Phys. Rev. A, 64, 063815 (2001).

[6] M. V.Fedorov, M. A. Efremov, P. A. Volkov, E. V. Moreva S. S. Straupe, S. P. Kulik, "Anisotropically and High Entanglement of Biphoton States Generated in Spontaneous Parametric Down-Conversion”, Phys. Rev. Lett., 99, 063901 (2007). 